\title{
memo: Spectacular developments during the last decade from the Section Editors' point of view
}

\author{
Silvia Hofer · Eberhard Gunsilius · István Láng • Vladimir Moiseyenko
}

Published online: 13 March 2017

(C) Springer-Verlag Wien 2017

\section{Spectacular developments in neuro-oncology during the last decade}

\section{Silvia Hofer, Switzerland}

The 2016 World Health Organization Classification of Tumors of the Central Nervous System introduced for the first time molecular parameters in addition to histology to define tumor entities. The new classification added recently recognized neoplasms, and deleted some entities, variants, and patterns that no longer have diagnostic and/or biological relevance. As such, diffuse infiltrating gliomas WHO II-IV of adults are divided into IDH-wild-type (wt) and IDH-mutant (mut) tumors. Mutant IDH-proteins synthesize an onco-metabolite (2-hydroxyglutarate) associated with the induction of a "CpG island DNA methylator phenotype" and a better prognosis. Loss of $1 p$ and $19 q$ (1p/19q co-deletion) defines oligodendroglial tumors associated with a better prognosis and better response to radiation therapy (RT) and chemotherapy. For routine use, three categories of diffuse glioma are proposed: (a) $\mathrm{IDH}_{\mathrm{mut}}, 1 \mathrm{p} / 19 \mathrm{q}$ co-deleted, WHO II or III; (b) $\mathrm{IDH}_{\mathrm{mut}}, 1 \mathrm{p} / 19 \mathrm{q}$ intact, WHO II or III, seldom IV; and (c) $\mathrm{IDH}_{\mathrm{wt}}$, WHO IV, seldom II or III.

\author{
S. Hofer $(\bowtie)$ \\ Section Editor Neurooncology, Lucerne, Switzerland \\ silvia.hofer@luks.ch \\ E. Gunsilius \\ Section Editor Haematology, Innsbruck, Austria \\ eberhard.gunsilius@i-med.ac.at \\ I. Láng \\ Section Editor Breast Cancer, Budapest, Hungary \\ lang@oncol.hu \\ V. Moiseyenko \\ Section Editor Gastrointestinal Cancer, St. Petersburg, Russia \\ moiseyenkov@gmail.com
}

Newly defined entities include among others: diffuse midline glioma, H3 K27M-mutant; RELA fusionpositive ependymoma; medulloblastoma, WNT-activated and SHH-activated. Other notable changes include the addition of brain invasion as a criterion for atypical meningioma and the introduction of a soft tissue-type grading system for the now combined entity of solitary fibrous tumor/hemangiopericytoma. The new classification will facilitate clinical, experimental, and epidemiological studies.

In the past 10 years, treatment of high-grade gliomas, especially glioblastoma (GBM) WHO IV, has not changed, despite several phase III trials with promising compounds and concepts. None of these proved to have added benefit to standard temozolomide (TMZ)-based postoperative radiochemotherapy, except for prolongation of overall survival by the application of tumor treating fields (TTF), a portable medical device that delivers low-intensity, intermediate-frequency $(200 \mathrm{kHz})$ alternating electric fields continuously via four transducer arrays placed on the shaved scalp. In the subgroup of elderly GBM patients, the previous standard of care (i.e., radiotherapy alone) has changed in the case of a methylated MGMT promoter status, and TMZ with or without radiation therapy (RT) seems superior to RT alone. Phase III trials with antiangiogenic agents all yielded negative findings, when overall survival is the endpoint, despite scientific rationale to use them since GBM are highly vascularized tumors and angiogenesis plays an important role in their biology.

Targeted agents often failed to give a signal in earlyphase trials for various suspected (e.g., molecular pathway redundancy) and yet unknown reasons. Immunotherapeutic concepts are under development.

For diffuse lower-grade gliomas (WHO II and III) there is now convincing evidence deriving from longterm outcome data in patients who were treated with 
RT plus chemotherapy, as compared with those who received postoperative RT alone. The treatment effect appeared to be largest, but not exclusively in patients with oligodendrogliomas and in tumors with $I D H 1$ $\mathrm{R} 132 \mathrm{H}$ mutations. Separation of the progression-free survival curves of the two treatment groups did not begin until years after randomization, the same trend of delayed separation was apparent in overall survival. Neurocognitive function preservation is an important issue for early combined treatment with expected long-term survival.

WHO I gliomas are usually well demarcated and cured by local therapies alone. The majority of pilocytic astrocytomas have been recognized as singlepathway diseases through activation of the mitogenactivated protein kinases (MAPK) driven by a BRAF proto-oncogene (KIAA1549-BRAF fusion gene).

\section{The most spectacular developments in hematol- ogy during the last decade}

\section{Eberhard Gunsilius, Austria}

It can be said that the field of hematology has never been as exciting as today. The treatment landscape has changed from a "one-size-fits-all" strategy to riskadapted treatment protocols for pathologically and clinically defined prognostic subgroups in the past decades, and today specific genetic signatures and the development of targeted therapies against welldefined antigens or kinases are the hallmark of the fascinating new era of personalized cancer therapy. Now we can define many hematologic malignancies by their genetic pattern, allowing us to allocate individual patients to the best treatment, i. e., to select patients most likely to respond to targeted therapy, and, on the other hand, those who are most likely not responding and therefore can be offered alternative therapies. Listing all of these achievements would go beyond the scope of an editorial.

Remarkable achievements in diagnostics and treatment have been made in virtually all hematologic neoplasms, such as molecular markers and therapies for myeloproliferative diseases (mutations in JAK-2 and calreticulin genes, JAK-2 inhibitors), novel monoclonal antibodies and small molecules targeting Bruton's tyrosine kinase, bcl-2 and PI3-kinase in chronic lymphocytic leukemia, and immunotoxins against CD30 in Hodgkin's lymphoma and particular lymphoma subtypes. Chronic myeloid leukemia has become a "curable" malignancy thanks to the development of potent tyrosine kinase inhibitors leading to the steady decrease of allogeneic stem cell transplantation for this leukemia. Impressive results can now be obtained by restoring the activity of the immune system by blocking inhibitory immune checkpoint molecules such as PD-1 molecules with nivolumab in advanced Hodgkin's disease. Sophisticated techniques such as real-time PCR, multicolor flow cytometry, next-generation sequencing, etc. for the monitoring of minimal residual disease are paving the way for tailored treatment strategies in individual patients.

Another success story is multiple myeloma. Owing to the development of new drugs such as proteasome inhibitors and immunomodulatory agents and, very recently, monoclonal antibodies and their implementation in modern treatment regimens, the survival of patients with myeloma has doubled within 10 years. Notably, most of the progress in this disease has been achieved in low-risk patients. Patients with high-risk disease, defined by distinct genetic alterations or clinically by early relapse after first-line therapy, still need better treatment options.

In the future, further advances in genomic profiling, new drug combinations, and chimeric antigen receptor T-cell gene therapy "out of the box" are likely to improve further the clinical outcomes for tens of thousands of patients.

However, as often happens in life, the flip side of the coin also has to be considered. Pricing is out of reach. The costs for modern anticancer drugs have skyrocketed in the past few years and can exceed $€ 100,000$ per patient per year. This financial burden may become unsustainable for health-care systems, even in Western countries. The increasingly soaring prices predict a gloomy prospect that for economic reasons "non-doctors" might dictate our daily work in the future.

\section{Drug shortages}

The withdrawal of alemtuzumab for the treatment of chronic lymphocytic leukemia from the market to prepare for a higher-priced relaunch of this drug aimed at multiple sclerosis has robbed a valuable treatment option for our patients, and the recurrent bottleneck in the supply of melphalan for autologous transplants is shameful.

However, in Austria, and in a few other countries in Europe, we are privileged in that we can offer our patients most of the novel drugs as early as they appear on the market, which is often years before doctors in other countries get access to newly registered drugs.

\section{Spectacular developments in breast cancer on- cology}

\section{István Láng, Hungary}

With 1,700,000 new cases diagnosed worldwide each year, breast cancer remains a leading cause of death among women, responsible for more than 500,000 deaths per year. Therefore, even small developments in its diagnosis and treatment are important and may save many lives.

During the past few decades, molecular profiling of breast cancer has become part of daily practice in developed countries, giving the chance for multidisciplinary oncology teams in dedicated breast can- 
cer centers to increase the efficacy of precision oncology by choosing tailored treatments for the patients. By better predicting early relapses, first-generation genomic signature tests such as Oncotype Dx and Mammaprint can help patients to avoid unnecessary adjuvant chemotherapy.

In hormone-sensitive disease the prolonged administration of adjuvant tamoxifen (10 years instead of 5 years) led to further improvements in mortality. Extending adjuvant aromatase-inhibitor treatment to 10 years for postmenopausal patients with hormonereceptor-positive early-stage breast cancer resulted in significantly longer disease-free survival and a lower incidence of contralateral breast cancer.

In high-risk premenopausal women with endocrine-sensitive breast cancer, the LHRH analogue triptorelin combined with exemestane is more effective in preventing recurrence than when combined with tamoxifen.

The development of endocrine resistance is one of the major problems in endocrine-responsive disease. Since PI3K/AKT/mTOR is the most frequently altered signal transduction pathway in this disease, intensive research has been underway to develop new agents targeting this pathway. The mTOR inhibitor everolimus combined with exemestane doubled progression-free survival and (although not statistically) prolonged overall survival leading to its approval by the FDA.

The pan-class PI3K inhibitor buparlisib has successfully overcome endocrine resistance and improved responses in clinical trials. Although toxicities like diarrhea and skin rash limit its routine clinical use, it has been approved by the FDA and it became the first pan-class PI3K inhibitor commercially available for the treatment of endocrine-resistant breast cancer.

Another promising way to overcome endocrine resistance is to target cyclin-dependent kinases 4 and 6 $(\mathrm{CDK} 4,6)$. The cyclin D1 gene is overexpressed in about $15 \%$ of breast cancer patients, and by interacting with CDK 4 and 6 it promotes cell proliferation. Thus inhibiting the $C D K 4,6$ pathways can help to fight endocrine resistance in hormone-receptor-positive breast cancer. The CDK 4, 6 inhibitors palbociclib and ribociclib have been recently approved by the FDA.

In hormone-receptor-negative young premenopausal women, the LHRH analogue goserelin can be used for ovarian protection and preservation of fertility during adjuvant chemotherapy.

Before the introduction of trastuzumab 16 years ago as the first anti-HER2-targeted therapy, HER2positive breast cancer (together with triple-negative disease) had the worst prognosis. Since the availability of anti-HER2 treatments, the prognosis has substantially improved: More patients with early-stage disease can be cured with (neo)adjuvant combined systemic therapy, and patients in advanced-stage disease can live much longer with their metastatic chronic disease. Using neoadjuvant chemotherapy combined with dual anti-HER2-targeted treatment (trastuzumab and pertuzumab) more earlystage HER2-positive patients can be cured. This is also true for the adjuvant use of chemotherapy plus trastuzumab. Further improvement can be achieved by adding adjuvant neratinib treatment following the standard adjuvant chemotherapy-trastuzumab combination. In metastatic HER2-positive breast cancer, the most effective first-line choice is chemotherapy with dual HER2 inhibition (trastuzumab and pertuzumab). Trastuzumab-emtansine (TDM-1), an antibody drug conjugate combining trastuzumab with chemotherapy, is the most effective second-/third-line therapy for advanced HER2-positive breast cancer. Although the cure rates of early-stage - and the survival of advanced HER2-positive - breast cancer patients have been substantially improved by these modern targeted treatments, because of their high price they are not readily available in countries with limited resources. The introduction of biosimilar agents such as MYL-14010 might help to solve this problem.

In most cases of triple-negative breast cancer, chemotherapy remains the only systemic treatment choice. In patients with BRCA mutations, therapy with PARP inhibitors gave encouraging results.

In triple-negative breast cancer and in patients expressing PDL-1, immunotherapy with anti-PD-1 and PDL-1 monoclonal antibodies could induce remarkable responses. These agents are not yet approved in breast cancer.

\section{Summary}

With the introduction of several new drugs targeting various specific targets, more early-stage breast cancer patients can be cured, and many advanced-stage patients can live much longer with their chronic disease with acceptable quality of life.

\section{Spectacular developments in colorectal cancer treatment}

\section{Vladimir Moiseyenko, Russia}

The past 10 years of consistent developments in the treatment of advanced colorectal cancer have led to a significant change in patient survival. While patients with metastatic colorectal cancer had a poor prognosis in the 1990s, today these patients can survive for more than 20 months. Many components of our routine clinical management influenced this change in survival. Multiple clinical trials introduced a shift in treatment strategy with a clear definition of treatment goals for each individual patient prior to treatment initiation. Consecutive and less toxic monotherapy for patients with unresectable, asymptomatic disease or an aggressive treatment approach with a wider use of surgery and metastasectomy in particular has al- 
lowed us to achieve an optimal survival advantage as far as the preservation of quality of life is concerned. The introduction of new treatment approaches such as maintenance therapy, for example, led to an increase in time to progression while preserving quality of life with low toxic regimens. Of course, the exploration of new molecular markers and the introduction of new therapeutic agents had a positive influence on the course of the disease.

During the past few years, a multidisciplinary treatment approach became an obligatory component of the management of colorectal cancer patients. For long it was known that patients with resectable liver metastases may achieve significant improvement in overall survival or even cure following complete resection of tumor lesions. During the past 10 years, this subgroup of patients was expanded to include those with borderline resectable disease. Tumor shrinkage following aggressive regimens combining chemotherapy and targeted agents in a significant proportion of patients allowed a resectable state to be achieved giving the chance for survival prolongation with a surgical approach.

Major advancements were made in our understanding of tumor heterogeneity. Today, colorectal cancer is a group of diseases with different prognoses and clinical behavior and of particular importance for clinicians with different treatment approaches required for optimal results. Although during all these years the backbone of therapy was based on chemotherapeutic agents and remained the same, we can make a clearer definition of the optimal population for targeted treatment based on predictive markers. KRAS and NRAs mutational status became a standard characteristic of the disease. And the WT status of these genes became a mandatory requirement for anti-EGFR monoclonal antibody use. This molecular-marker-based selection helped us achieve longer survival in patients with unresectable disease and to broaden the group of patients undergoing complete tumor resections.

Analysis of the prognostic and predictive tumor markers in large patient series elucidated multiple clinically distinct groups requiring different treatment approaches. The negative prognostic value of BRAF mutations in a metastatic setting, for example, translated into the more aggressive treatment approach for patients whose tumors harbor these aberrations. Surprisingly, tumors with MSI were shown to be exclusively sensitive to a novel type of systemic treatment - PD-1 inhibitors - presumably due to the high mutational burden leading to high immunogenicity, which gives a chance for a significantly longer survival in some patients.

During the past decade, novel complex methods of whole-genome molecular analysis were introduced into clinical oncology. We are now in the middle of a process of subtyping tumors arising in the colon into several types based on their whole-genome molecular portrait. In this new paradigm, particular molecular alterations that were shown to be of clinical importance might have different and even opposite effects on clinical behavior depending on the molecular context. Of particular interest we should mention one of the latest observations of the lower efficacy of antiEGFR agents in right-side colon tumors even without RAS mutations being closely related to the different molecular pathophysiology of tumors arising throughout the colon.

One of the most prominent recent developments is the realization of the prophylactic value of aspirin for colon cancer, which is potentially able to impact on cancer incidence on the population level.

Thus, major steps have been taken during the past decade in fundamental and clinical scientific research on colorectal cancer, but much more is to be done in the future in order to improve our patients' well-being.

Conflict of interest S. Hofer, E. Gunsilius, I. Láng, and V. Moiseyenko declare that they have no competing interests.

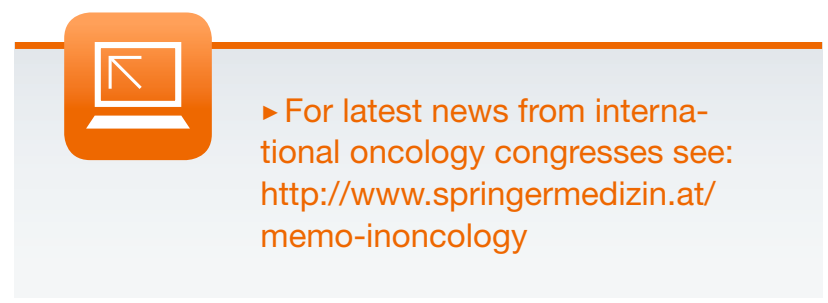

